\title{
QUEENS COLLEGE RADIOCARBON MEASUREMENTS III
}

\author{
RICHARD PARDI and E R NEWMAN
}

Radiocarbon Laboratory, Queens College, City University of New York Flushing, New York 11367

This list contains analyses completed between January, 1976 and April, 1977. Details of laboratory operation are contained in our first list ( $R, 1975, v 18, p$ 205). Samples submitted for analysis are reviewed by a committee consisting of W DeBoer, E Hansen, Anthropology; L Marcus, Biology; W S Newman, D L Thurber, Earth and Environmental Sciences; and Richard Pardi, Radiocarbon Laboratory.

All results are based on the conventional half-life of ${ }^{14} \mathrm{C}, i e, 5568 \pm$ 30 years. Results are $1 \delta$, based on the combined statistical counting error of the sample, background and standard. ${ }^{12} \mathrm{C} /{ }^{13} \mathrm{C}$ measurements and corrections have not been made for these samples.

Samples sub-labeled with letters are re-analyses of identical or equivalent samples. Those sub-labeled with numbers are repeat or duplicate counts on split samples of prepared benzene, unless otherwise noted.

ACKNOWLEDGMENTS

Sue Coughlin, Don Haarmann, and C Perdomo assisted in sample preparation. We wish to thank D H Speidel for his continued support.

\section{Sea level series}

\section{GEOLOGIC SAMPLES}

Peats (basal) coll and subm by W S Newman and L Cinquemani, Dept Earth Environmental Sci, Queens Coll, Oscawana I Tidal Marsh, Hudson R, New York (41 $\left.11^{\circ} 45^{\prime \prime} \mathrm{N}, 73^{\circ} 55^{\prime} 50^{\prime \prime} \mathrm{W}\right)$; Constitution I Tidal Marsh, Hudson R, New York $\left(41^{\circ} 23,5 \mathrm{~N}, 73^{\circ} 58^{\prime} \mathrm{W}\right)$; Piermont Tidal Marsh, Hudson R, New York $\left(41^{\circ} 1^{\prime} 30^{\prime \prime} \mathrm{N}, 73^{\circ} 54^{\prime} \mathrm{W}\right)$; Pelham Bay Park, Bronx, New York $\left(40^{\circ} 52.1^{\prime} \mathrm{N}, 73^{\circ} 47.6^{\prime} \mathrm{W}\right)$; Ring Meadow, Iona I., Hudson R, New York (41 $\left.11^{\circ} 30^{\prime \prime} \mathrm{N}, 73^{\circ} 58^{\prime} 40^{\prime \prime} \mathrm{W}\right)$. All samples treated with hot dilute $\mathrm{HCl}$.

QC-221A. Oscawana I, 7.5m

$5150 \pm 210$

Depth 7.5m below MHW.

QC-221B. Oscawana I, 6.8m

$4570 \pm 120$

Depth $6.8 \mathrm{~m}$ below MHW.

QC-228. Oscawana $I, 2.7 \mathrm{~m}$

$1870 \pm 90$

Depth $2.7 \mathrm{~m}$ below MHW.

QC-264. Oscawana I, $7 \mathrm{~m}$

$4500 \pm 100$

Depth $7 \mathrm{~m}$ below MHW.

QC-189. Constitution I, $9.45 \mathrm{~m}$

$5900 \pm 300$

Depth $9.45 \mathrm{~m}$ below MHW. 
QC-226. Constitution I, 3.9m

$2320 \pm 100$

Depth $3.9 \mathrm{~m}$ below MHW.

QC-227. Constitution I, 7.7m

$4230 \pm 120$

Depth $7.7 \mathrm{~m}$ below MHW.

QC-276. Constitution I, 6.15m

$4110 \pm 100$

Depth $6.15 \mathrm{~m}$ below MHW.

QC-262. Piermont, 5.05m

$3460 \pm 100$

Depth $5.05 \mathrm{~m}$ below MHW.

QC-261. Piermont, 8.54m

$4610 \pm 120$

Depth $8.54 \mathrm{~m}$ below MHW.

QC-211. Piermont, 3m

$2300 \pm 160$

Depth 3m below MHW.

QC-295. Pelham Bay, 2.25m

$1800 \pm 90$

Depth 2.25m below MHW.

QC-274. Ring Meadow, $4.6 \mathrm{~m}$

$3610 \pm 120$

Depth $4.6 \mathrm{~m}$ below MHW.

QC-186. Ring Meadow, 10.75m

$3940 \pm 140$

Depth $10.75 \mathrm{~m}$ below MHW. Comment (WSN): further field check disclosed that tidal marsh at this point extends to greater depth.

QC-187. Ring Meadow, $4.75 \mathrm{~m}$

$3800 \pm 160$

Depth $4.75 \mathrm{~m}$ below MHW.

\section{Long Island sea level series}

Peats (basal) coll and subm by M Rampino, Goddard Inst for Space Studies, Wantagh, Nassau Co, Long Island, New York $\left(40^{\circ}\right.$ $\left.39^{\prime} \mathrm{N}, 73^{\circ} 31^{\prime \prime} \mathrm{W}\right)$. Back barrier area, Cedar Beach Suffolk Co, New York $\left(40^{\circ} 37^{\prime} \mathrm{N}, 73^{\circ} 23^{\prime} \mathrm{W}\right)$.

General Comment (MR): depth estimate error may be as great as $.15 \mathrm{~m}$ due to compaction.

QC-314. Cedar Beach, A76-32 $\quad 5060 \pm 120$

Depth $10.1 \mathrm{~m}$ below MSL.

QC-315. Wantagh, Cl-BP $1020 \pm 100$

Depth $1.12 \mathrm{~m}$ below MSL.

QC-316. Wantagh, C3-BP $\quad 300 \pm 90$

Depth $0.27 \mathrm{~m}$ below MSL.

\section{Bergen County mastodon series}

Bone, wood, and peat samples from a mastodon site, Bergen Co, New Jersey $\left(40^{\circ} 59^{\prime} \mathrm{N}, 73^{\circ} 57^{\prime} \mathrm{W}\right)$. Coll and subm by S Averill, 8 Willow Brook Rd, Hillsdale, New Jersey. 
QC-141. \#5

$5220 \pm 120$

Dentine from mastodon, depth ca $1.4 \mathrm{~m}$ below surface. Sample had been shellaced. An attempt was made to remove the shellac via soxhlet extraction in hot ethanol for 3 to 4 days. Comment (SA): previous date I-6828: $9125 \pm 150$ on lower stratigraphic level.

QC-142. \#6

Bone from mastodon. Also shellaced; treated same as QC-141.

QC-144. \#8

$5080 \pm 160$

Peat from above mastodon, depth ca $1.2 \mathrm{~m}$ below surface. Sample diluted with "dead" benzene.

QC-296. \#9

$12,130 \pm 210$

Peat from ca $1.8 \mathrm{~m}$ below surface. Large sample was sieved through 40-mesh screen and treated with hot dilute $\mathrm{HCl}$ and $\mathrm{KOH}$. Comment (SA): from 20 to $28 \mathrm{~cm}$ above thin tan lacustrine clay over coarse outwash of most recent glaciation. Peat from which mastodon, dated QC-141 and -142 , was removed.

QC-297. \#10

$12,820 \pm 200$

Peat treated same as QC-296. Comment (SA): from 5 to $15 \mathrm{~cm}$ above tan lacustrine clay.

\section{Malaspina Glacier series}

Samples coll and subm by $\mathrm{J} \mathrm{H}$ Hartshorn, Univ Massachusetts, from Malaspina Glacier, Alaska.

QC-160. MAL 15-70

$650 \pm 80$

Wood (Sitkagi Spruce?) torn up and embedded in till in front of glacier $\left(59^{\circ} 44^{\prime} \mathrm{N}, 140^{\circ} 30^{\prime} \mathrm{W}\right)$. Comment $(\mathrm{JHH})$ : till is subglacial; date gives age of last readvance of Malaspina Glacier to sea at Sitkagi Bluffs.

QC-161. MAL 26-70

$2770 \pm 90$

Wood embedded in a superglacial esker (gravel) emerging from beneath glacier $\left(59^{\circ} 50^{\prime} \mathrm{N}, 140^{\circ} 4^{\prime} \mathrm{W}\right)$. Comment $(\mathrm{JHH})$ : date to be used in recreating history of advance and retreat of Malaspina Glacier. Location of wood in englacial (now superglacial) esker is unusual.

QC-162. MAL 28-70

$3630 \pm 130$

Shell coll from surface of esker recently emerged from ice $\left(59^{\circ} 47^{\prime}\right.$ $\left.\mathrm{N}, 140^{\circ} 10^{\prime} \mathrm{W}\right)$. Comment $(\mathrm{JHH})$ : in 1951 , esker was beginning to emerge from beneath ice. Shells date from period of ice recession when sea was far $\mathrm{N}$ of present limits. When ice readvanced, shells were incorporated in till or ice and then treated as gravel by meltwater streams.

\section{Almond (Kent) Glacier series}

Dates used to reconstruct history and limits of Almond (Kent) Glacier. Coll and subm by E $\mathrm{H}$ Muller and P Willette, Dept Geol, Syracuse Univ. 
QC-232. PW-75-264

Peat with wood fragments and cones, Sargent's Peat Bog, New York $\left(42^{\circ} 17^{\prime} 32^{\prime \prime} \mathrm{N}, 78^{\circ} 11^{\prime} 0^{\prime \prime} \mathrm{W}\right)$, alt ca $450 \mathrm{~m}$, depth $3 \mathrm{~m}$ below bog surface. Comment (EHM): date will provide upper limit for Kent glaciation in Genesee Valley.

QC-233. PW-75-38

$6270 \pm 340$

Wood fragments contained in stratified, well-sorted, unoxidized silt, probably of alluvial origin, Estabrook site, Allegheny Co, New York $\left(42^{\circ} 25^{\prime} 14^{\prime \prime} \mathrm{N}, 78^{\circ} 8^{\prime} 20^{\prime \prime} \mathrm{W}\right)$, alt ca $370 \mathrm{~m}$, depth $4.5 \mathrm{~m}$ below surface. Very small sample, diluted with "dead" benzene (date too young). Comment (EHM): post-dates Almond (Kent) Glacier, related to early phase of erosional terrace development in reach of Genesee Stream upstream from Portageville and Letchworth Canyon.

QC-238. EM60-185C

25,450

$+6680$

Wood fragments contained in silty, sparsely to moderately stony, gray till, unconformably overlain by modern alluvial-fan gravels, Rush Creek, $600 \mathrm{~m}$ E of West Hill Rd, Allen Township, Fillmore Quad, Livingston City New York ( $\left.42^{\circ} 25^{\prime} 50^{\prime \prime} \mathrm{N}, 78^{\circ} 3^{\prime} 10^{\prime \prime} \mathrm{W}\right)$, alt ca $420 \mathrm{~m}, 60 \mathrm{~cm}$ above stream. Comment (EHM): till predates Kent glaciation; is 1st such date in central New York. Analysis of 2nd, larger wood sample coll in vicinity of QC-233.

QC-263. PW-75-38-2

Repeat analysis on larger sample of same wood as QC-233. Comment (EHM): 2nd date agrees well with field relationships and provides a datum point in downcutting which followed Lake Belfast-Fillmore and was controlled by bedrock incision directly $\mathrm{N}$ of Portageville.

Adak I series

Peat and fine organic sediments from drained pond (Black, 1975), Adak I., Alaska ( $\left.51^{\circ} 54^{\prime} 54^{\prime \prime} \mathrm{N}, 176^{\circ} 37^{\prime} 56^{\prime \prime} \mathrm{W}\right)$. Coll and subm by R H Black, Univ Connecticut, Storrs. QG dates are all on $>40$-mesh size fraction, treated with hot, dilute $\mathrm{HCl}$ and $\mathrm{NaOH}$.

General Comment (RFB): some fine organic sediments probably washed and blown in. Geochron dates on fraction $<80$-mesh.

QC-204A. 75A-182

$3830 \pm 110$

Depth 79 to $89 \mathrm{~cm}$ below surface. Treated with hot, concentrated $\mathrm{HCl}$ and NaCl04. Previously dated GX-4028: $3440 \pm 165$. Comment (RFB): most reliable date of series; from above Sandwich Ash.

QC-205. 75A-183

$3770 \pm 100$

Depth 99 to $109 \mathrm{~cm}$ below surface. Previously dated GX-3970: $4390 \pm 150$. Comment (RFB): from below Sandwich Ash. 
QC-206B. 75A-184.

$5110 \pm 200$

Depth 127 to $137 \mathrm{~cm}$ below surface. Previously dated GX-3971: $6800 \pm 175$. Comment (RFB): from above Intermediate Ash.

QC-207. 75A-185

$3040 \pm 370$

Small sample severely diluted with "dead" benzene (date too young), depth 145 to $155 \mathrm{~cm}$ below surface; previously dated GX-3972: $5705 \pm 200$. Comment (RFB): from below Intermediate Ash.

QC-208. 75A-186

$6660 \pm 90$

Depth 181 to $191 \mathrm{~cm}$ below surface, previously dated GX-4029: $8310 \pm 265$. Comment $(\mathrm{RFB})$ : from above Main ash, Geochron date too old.

QC-244. 76A-5

$4460 \pm 180$ 184.

Comment (RFB): from above Intermediate Ash, equivalent to $75 \mathrm{~A}$ -

QC-245. 75A-6

$6960 \pm 100$ 185.

Comment (RFB): from below Intermediate Ash, equivalent to $75 \mathrm{~A}$ -

\section{Deep-Sea Core series}

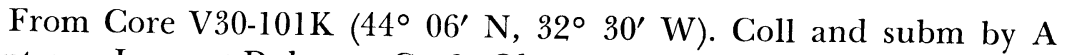
McIntyre, Lamont-Doherty Geol Observatory, Palisades, New York. General Comment (AMcI): results on QC-247, QC-248, and QC-249 were judged too old by about 10,000 yr each. QC-317 was run as a check, result agrees with Lamont date on split of same sample, 18,590 \pm 800 (L-1447B). Other dates on same core are QC-198 at 16,360 \pm 220 years, from depth of 63.5 to $71.5 \mathrm{~cm}, \mathrm{GX}-4491$ at $13,520 \pm 410 \mathrm{yr}$ from depth $32.5 \mathrm{~cm}$, and GX-4492 at $16,760 \pm 515$ years from depth $39 \mathrm{~cm}$. No explanation has been found for apparently anomalous results on the three deepest samples.

QC-246. V30-101K, 25cm

Ca $66 \%$ CaCO3.

QC-247. V30-101K, $40 \mathrm{~cm}$

$10,850 \pm 280$

Ca $45 \%$ CaCO3.

QC-248. V30-101K, 50cm

Ca $41 \%$ CaCO3.

QC-249. V30-101K, $55 \mathrm{~cm}$

Ca $45 \%$ CaCO3.

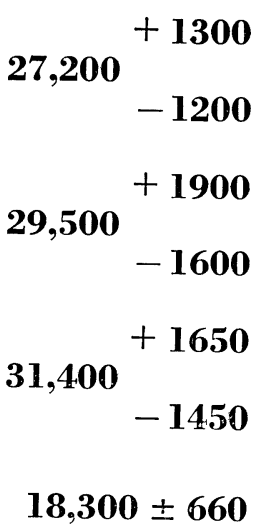

QC-317. V30-101K, $46.8 \mathrm{~cm}$

Ca $45 \%$ CaCO3. 
QC-112. \#12

Charcoal from hearth on much used camping floor, Dogan Pt, Haverstraw, New York $\left(41^{\circ} 14^{\prime} 10^{\prime \prime} \mathrm{N}, 73^{\circ} 56^{\prime} 50^{\prime \prime} \mathrm{W}\right)$. Depth $40 \mathrm{~cm}$ below surface. Coll and subm by L A Brennan, Briarcliff Coll, Ossining, New York. Comment (LAB): sample too young; evidence of much camping and many fills; charcoal subm along with soil matrix. Assoc with small stemmed (Taconic) points, Perkiomen, small side-notched points (Twombly), and distinctive industry probably related to Susquehanna. There was no pottery.

\section{Pipins Rock series}

Charcoal and shell (C virginica) from Pipins Rock site $\left(41^{\circ} 10^{\prime} \mathrm{N}\right.$, $\left.73^{\circ} 52^{\prime} \mathrm{W}\right)$. Coll and subm by L A Brennan.

QC-225. \#1 and \#2

$\mathbf{3 3 7 0} \pm \mathbf{1 7 0}$

Charcoal from apparent fire pit extending downward in basal sands, depth 60 to $70 \mathrm{~cm}$ below surface. Sample dated is composite of 3 small charcoal samples from same region. Comment (LAB): fire pit is within $15 \mathrm{~cm}$ of shell deposit and is assoc with small series of excurvate sided triangular points with basal flute flake removed.

QC-224. 13E34S

$4400 \pm 100$

Shell from same level as QC-225, in undisturbed sand $25 \mathrm{~cm}$ from hearth. Comment (LAB): Taconic stemmed point found in vicinity; $4400 \mathrm{BP}$ is good date for Taconic series here.

QC-239. S Side Midden \# 1

$4370 \pm 90$

Small oyster shells, depth $56 \mathrm{~cm}$ below surface.

QC-240. S Side Midden \#2

$4940 \pm 100$

Three large oyster shell valves, depth $61 \mathrm{~cm}$ below surface.

QC-241. S Side Midden \#3

$2480 \pm 340$

Charcoal from small pit hearth in midden, depth $56 \mathrm{~cm}$ below surface, assoc with QC-243.

QC-243. S Side Midden \#5

$4490 \pm 90$ surface.

Oyster shells from around and beneath hearth, depth $51 \mathrm{~cm}$ below

\section{QC-270. S Side Midden \#6}

1) $4600 \pm 80$

2) $4700 \pm 90$

Small shells from yellow sand-clay basement under midden, depth 56 to $61 \mathrm{~cm}$ below surface.

\section{Spruce Swamp series}

Spruce Swamp site, East Norwalk, Connecticut $\left(41^{\circ} 5^{\prime} 16^{\prime \prime} \mathrm{N}, 73^{\circ}\right.$ $\left.23^{\prime} 18^{\prime \prime} \mathrm{W}\right)$. Coll and subm by E L Claypool, SW Connecticut Archaeol Comm, 23 Plymouth Rd, Stamford, Connecticut 06906. 
QC-217. SS-1

Shell ( $C$ virginica), depth $80 \mathrm{~cm}$ below MSL. Comment (ELC): from Feature \#1, earth wholly enclosed in glacial sand and gravel. Sample dates earliest known aboriginal occupation at Spruce Swamp site.

QC-231. SS-2

$130 \pm 90$

Acorns (Quercus sp), uncharred and decomposed, from storage pit (Feature \#15), in black soil midden between shell midden and glacial deposits containing $C$ virginica shells dated at QC-217. Depth $6 \mathrm{~cm}$ above to $50 \mathrm{~cm}$ below MSL. Comment (ELG): dates only known acorn storage pit in New England, estimated age was $2500 \mathrm{yr}$ BP, based on sea level curve and relationship to QC-217. Possible sources of contamination: salt water, sewage, sludge, marine engine waste from adjacent marina, and heavy penetration of roots.

QC-273. SS-3

$\mathbf{8 0} \pm \mathbf{5 0}$

Twigs (tree sp unidentified) from lining of Feature \#15, acorn storage pit, uncarbonized and decomposed.

QC-298. SS-4

$750 \pm 90$

Wood (sp unknown) from either remains of prehistoric post or tree root. Solid wood, not decomposed, showing possible rings, depth 45 to $72 \mathrm{~cm}$ below MSL. Comment (ELC): adjacent to Feature \#15. Same contamination possibilities as QC-231.

QC-318. SS-5

$610 \pm 350$

Charcoal assoc with pottery from above Feature \#15, depth $18 \mathrm{~cm}$ below MSL. Very small sample, diluted with "dead" benzene. Comment (ELC): date is minimum for Feature \#15.

\section{QC-332. SS-6}

$1050 \pm 80$

Shell (Venus mercenaria), depth $23 \mathrm{~cm}$ below surface, alt $7 \mathrm{~cm}$ above MSL. Result suggests that dates on acorns, QC-231, and twigs, QC-273, from storage pit (Feature \#15) are too young, since, even correcting for expected apparent age of recent shell material (Mangerud, 1972), shells should not be younger than ca 850 yr. Comment (ELC): from Stratum 2 above acorn storage pit.

QC-307/8. \#1 and \#2

$2850 \pm 220$

Bone fragments from hearth, rockshelter, Brewster, New York $\left(41^{\circ} 22.5^{\prime} \mathrm{N}, 73^{\circ} 30^{\prime} \mathrm{W}\right)$, depth 1.3 to $1.4 \mathrm{~cm}$ below surface; coll and subm by R C Thompkins, Poughkeepsie. Combined sample was very small, and was diluted with "dead" benzene. Comment (RCT): sample assoc with Palmer-like projectile point.

\section{Shawnee-Minisink site}

From flood plain of Delaware R at confluence with Brodford Creek near Stroudsburg, Pennsylvania $\left(50^{\circ} 59^{\prime} 0^{\prime \prime} \mathrm{N}, 75^{\circ} 8^{\prime} \mathrm{W}\right)$. Coll and subm by $\mathrm{C}$ W McNett, American Univ. 
QC-250.

$$
1990 \pm 80
$$

Charcoal from Sqs 25 and 25, Level 04, depth $60 \mathrm{~cm}$ below surface.

QC-259.

$1610 \pm 100$

Charcoal from Sqs 5 and 9, Level 08, depth $85 \mathrm{~cm}$ below surface.

QC-157/8. S6E66-1 and S2W6-19

$1040 \pm 120$

Charred wood from possible fire pit (Feature 1), Mason I II site (18M013), Maryland (39 $\left.10^{\prime} 50-53^{\prime \prime} \mathrm{N}, 77^{\circ} 29^{\prime} 45-55^{\prime \prime} \mathrm{W}\right)$, depth 47 to $68 \mathrm{~cm}$ below surface. Coll and subm by K Franklin, American Univ. Comment (KF): date is additional for Montgomery focus-related pottery type in Potomac Valley and evidence to suggest its temporal relationship to Luray focus.
QC-222. Sesuit Harbor
1) $1790 \pm 120$

Wood, white cedar (Chamaecyparis thyoides) from possible site, Sesuit Harbor, Massachusetts (41 $\left.45^{\prime} 10^{\prime \prime} \mathrm{N}, 70^{\circ} 10^{\prime} 21^{\prime \prime} \mathrm{W}\right)$, coll and subm by $\mathrm{S}$ Coughlin, Queens Coll, and R Prescott, Cape Cod Mus Nat Hist, Brewster, Massachusetts. Comment (SC): sample from former freshwater swamp now located in mire intertidal zone. Area subject to daily tidal flooding.

\section{South Windsor series}

Site \#6Ht89, South Windsor, Connecticut $\left(45^{\circ} 50^{\prime} \mathrm{W}, 72^{\circ} 37^{\prime} 30^{\prime \prime} \mathrm{N}\right)$. Coll and subm by $\mathrm{K}$ McBride and $\mathrm{W}$ Stinson, Univ Connecticut.

QC-301. N5W5/4

$3510 \pm 140$

Charred wood from hearth overlain with ca $1.1 \mathrm{~m}$ floodplain silt loam, alt 7.6m ASL.

\section{Gatecliff Shelter series}

Gatecliff Shelter, Nevada $\left(39^{\circ} 00^{\prime} \mathrm{N}, 116^{\circ} 47^{\prime} \mathrm{W}\right)$; coll and subm by D H Thomas, American Mus Nat Hist, New York.

QC-287. GU-9, \#402

Charcoal, Unit X, Feature 1, depth $300 \mathrm{~cm}$.

QC-288. GU-9, \#409

Charcoal, Unit II, Feature 7, depth $293 \mathrm{~cm}$.

QC-289. GU 4-74, \#416

Charcoal, Unit XX, Feature 1, depth $594 \mathrm{~cm}$.

QC-290. GU 6-74, \#418

1) $2900 \pm 90$

2) $3130 \pm 90$

Charcoal, Unit XXIII, depth $592 \mathrm{~cm}$.

QC-291. GU 1-74, \#419

$7080 \pm 680$

Charcoal, Unit XX, depth $535 \mathrm{~cm}$. Very small sample, severely diluted with "dead" benzene. Comment (DHT): despite large statistical error, date is roughly as expected. 
QC-292. GU 6-74, \#421

Charcoal, Unit X.

$4140 \pm 130$

QC-293. GU 4-74, \#422

Charcoal, Unit XXIII.

QC-294. B-5, \#441

$5100 \pm 100$

Charcoal, from Triple $T$ shelter, West Northum 2) $\mathbf{5 4 1 0} \pm \mathbf{2 7 0}$ Nye Co, Nevada $\left(39^{\circ} 00^{\prime} \mathrm{N}, 116^{\circ} 55^{\prime} \mathrm{W}\right)$, alt +2079 Coll and subm by $\mathrm{H}$ A Thomas.

\section{QC-271. Excavation 1, Level 7}

A) $2650 \pm 80$

B) $2805 \pm 130$

Charcoal from hearth (Feature \#1), Parmana village site, Guarico State, Venezuela $\left(8^{\circ} \mathrm{N}, 66^{\circ} \mathrm{W}\right)$ depth 125 to $145 \mathrm{~cm}$ below surface. B pretreated, as in Haynes (1966). Coll and subm by A Roosevelt, Mus American Indian. Comment (AR): possible contamination from rootlets and lignite. Sample from Early Corozal phase.

\section{Ronquin Sombra series}

Ronquin sombra site, Dist Infante, Guarico State, Venezuela $\left(8^{\circ} \mathrm{N}\right.$, $\left.66^{\circ} \mathrm{W}\right)$. Coll and subm by A Roosevelt.

QC-311A. Exeavation 8, Level 7

$1170 \pm 100$

Charcoal, depth 95 to $110 \mathrm{~cm}$ below surface. Comment (AR): from Ronquin phase.

QC-311B. Excavation 8, Level 8

$1450 \pm 70$

Charcoal, depth 110 to $125 \mathrm{~cm}$ below surface. Comment (AR): from Ronquin phase.

QC-327. Excavation 9, Level 9

Charcoal, depth 137 to $147 \mathrm{~cm}$ below surface.

$1300 \pm 90$

\section{Corozal series}

Corozal site, Guarico State, Venezuela $\left(8^{\circ} \mathrm{N}, 66^{\circ} \mathrm{W}\right), 3 \mathrm{~km}$ NE of Parmana village. Site in tropical gallery forest. Coll and subm by $\mathrm{A}$ Roosevelt.

\section{QC-272. Excavation 2, Level 20}

A) $23,900 \pm 650$

B) $24,700 \pm 1250$

Lignite, depth 147 to $160 \mathrm{~cm}$ below surface. B bleached with perchlorate after regular pretreatment. Comment (AR): from Early Corozal phase.

\section{QC-275. Excavation 2, Level 20}

A) $25,400 \pm 650$

Lignite, B treated with perchlorate $\quad$ B) $\mathbf{2 4 , 7 0 0} \pm 200$

\section{QC-309. Excavation 3, Level 4}

$860+70$

Charcoal, shiny and not very dense, depth 30 to $53 \mathrm{~cm}$ below surface. Some rootlets removed. Comment (AR): from Late Camoruco phase. 


\section{QC-310. Excavation 3, Level 10}

A 1) $510 \pm 70$

2) $540 \pm 80$

B 1) $410 \pm 80$

2) $340 \pm 90$

Charcoal, depth 130 to $143 \mathrm{~cm}$ below surface. B separately coll equivalent sample. Comment (AR): from Middle Camoruco phase.

QC-319. Excavation 2, Level 7

$\mathbf{7 2 0} \pm \mathbf{8 0}$

Charcoal, depth 112 to $138 \mathrm{~cm}$ below surface. Comment (AR): from Middle to Late Camoruco phase.

QC-320. Excavation 2, Level 8

$$
260 \pm 80
$$

Charcoal, depth 138 to $153 \mathrm{~cm}$ below surface. Comment (AR): from Middle Camoruco phase.

QC-321. Excavation 2, Level 9

$410 \pm 90$

Charcoal, depth 153 to $167 \mathrm{~cm}$ below surface. Comment (AR): from Early Camoruco phase.

QC-322. Excavation 2, Level 12

$820 \pm 90$

Charcoal, depth 210 to $230 \mathrm{~cm}$ below surface. Comment (AR): from Late Corozal period.

QC-323. Excavation 2, Level 14

Charcoal, depth 250 to $275 \mathrm{~cm}$ below surface. Comment (AR): from Middle Corozal phase.

QC-324A. Excavation 3, Level 11A

$460 \pm 80$

Charcoal, depth 145 to $153 \mathrm{~cm}$ below surface. Comment (AR): from Early to Middle Camoruco phase.

QC-325. Excavation 3, Level 12A

$830 \pm 90$

Charcoal, depth 153 to $170 \mathrm{~cm}$ below surface. Comment (AR): from Early Camoruco to Late Corozal phase.

QC-326. Excavation 3, Level $15 \quad 1200 \pm 90$

Charcoal, depth 198 to $210 \mathrm{~cm}$ below surface. Comment (AR): from Early Camoruco to Late Corozal phase.

\section{QC-335. Excavation 2, Level 20}
1) $23,080+910$
2) $22,420+820$
3) $\mathbf{3 7 2 0} \pm \mathbf{2 7 0}$

Lignite. Dates 1) and 2) are duplicate runs on same pretreated sample. Date 3) is date on extracted humic acids from 1) and 2).

\section{Los Mangos series}

Los Mangos site, Infante Dist Guarico State, Venezuela $\left(8^{\circ} \mathrm{N}\right.$, $\left.66^{\circ} \mathrm{W}\right), 100 \mathrm{~m}$ SE Parmana village. Coll and subm by A Roosevelt. 


\section{QC-312A. Excavation 1, Level 17}

A) $340 \pm 80$

$\begin{array}{ll}\text { Charcoal, depth } 260 & \text { B) } 900 \pm 90\end{array}$ equivalent to A. B small to $280 \mathrm{~cm}$ below surface. B stratigraphically (AR): from Middle to Late Corozal phase. with "dead" benzene. Comment

\section{QC-313A. Excavation 1, Level 18}

A) $1170 \pm 90$

Charcoal, depth 280 to $300 \mathrm{~cm}$ below surface. A stratigraphically equivalent to B. Comment (AR): from Middle to Late Corozal phase.

\section{REFERENCES}

Black, R F, 1975, Late Quaternary geomorphic processes: Effects on the ancient Aleuts of Unmak Island in the Aleutians: Arctic, v 28, p 159-169. Haynes, C V, Jr, 1966, Radiocarbon samples: Chemical removal of plant contaminants:
Science, v 151, p 1391-1392.

Mangerud, J, 1972, Radiocarbon dating of marine shells, including a discussion of apparent age of recent shells from Norway: Boreas, v 1, p 143-172.

Pardi, R R, 1975, Queens College radiocarbon measurements I: Radiocarbon, v 18,
p 205-209. 